$\left.\begin{array}{c}\text { Kalpa Publications in Engineering } \\ \text { Volume 3, 2020, Pages 11-18 } \\ \text { Proceedings of International Sym- } \\ \text { posium on Applied Science 2019 }\end{array}\right)$ Kalpel

\title{
Development of three-dimensional physiological function imaging of biological body by transillumination imaging using near infrared light - preliminary research -
}

\author{
Ngoc An Dang Nguyen ${ }^{1,3}$, Phuong Anh Bui ${ }^{2}$, Anh Tu Tran ${ }^{1}$, Trung An \\ Dang Nguyen ${ }^{3}$, Van Chinh Nguyen ${ }^{3}$, Koichi Shimizu ${ }^{4}$, and Trung Nghia Tran ${ }^{1}$ \\ ${ }^{1}$ Hochiminh City University of Technology (VNUHCM), Ho Chi Minh City, Vietnam \\ ${ }^{2}$ Vietnam National University Ho Chi Minh City, Ho Chi Minh City, Vietnam \\ ${ }^{3}$ University of Medicine and Pharmacy Ho Chi Minh City, Ho Chi Minh City, Vietnam \\ ${ }^{4}$ Waseda University, Kitakyushu, Japan \\ dnnan.sdh19@hcmut.edu.vn, ttnghia@hcmut.edu.vn
}

\begin{abstract}
In biological tissue, there are different kinds of endogenous chromophores. Their absorption spectra in the optical range are sensitive to the physiological change of the animal body. In the near-infrared region (700-1200 nm wavelength), hemoglobin has a characteristic absorption spectrum which is dependent on its redox state. Therefore, the functional information inside the animal body could be obtained noninvasively by measuring the transmitted light. By detecting the change of the absorption characteristics of the animal body, the functional change inside the body can be detected in a two-dimensional transillumination image. In this paper, we propose a preliminary research on development a novel imaging modality of biological body parts. Using the two-dimensional images obtained in many different orientations, three-dimensional physiological function imaging of biological body by transillumination could be expected. This paper presents a preliminary result in the experiment to show the feasibility of this technique.
\end{abstract}

Key word: Transillumination, functional imaging, near-infrared, blood vessel, 3D reconstruction, scattering suppression, deconvolution, point-spread-function (PSF). 


\section{Introduction}

The importance of transillumination imaging for blood vessels using near-infrared (NIR) light for medical and biometrics fields has been recognized [1-2]. In previous studies of our groups, the active studies were conducted on the transillumination imaging for biological bodies [3-5]. Using a nearinfrared (NIR) light with 700-1200 $\mathrm{nm}$ wavelength, we could visualize the macroscopic internal structure of an animal body (kidney, liver ...), the blood vessels inside the human body parts [4-5]. Even with a simple system (light-emitting diode (LED)'s array and low-cost charge-coupled device (CCD) camera), we could obtain the blood vessel transillumination image of the human forearm. We successfully developed a technique using the deconvolution with a fluorescent depth-dependent point spread function to suppress the scattering effect and verified their feasibility in experiments. We also developed an optical computed tomography (optical CT) modality using near-infrared light (NIR) for a small animal body. With obtained projection images from many orientations after suppressing scattering effect, we can reconstruct a three dimensional (3D) image using the common filtered-backprojection technique [4].

In recent years, some devices have been developed by other groups using infrared light to view the blood vessel as VeinLite_www.veinlite.com or early detection of breast abnormalities as BreastLight www.breastlightsouthafrica.co.za. However, the image is severely blurred due to the strong scattering in body tissue.

Several recent studies have also shown that an optical diagnosis can help predict the ability to respond to breast cancer chemotherapy before conducting actual chemotherapy [6-7]. This helps patients know early that they will not respond to chemotherapy and allows doctors to decide to change their treatment regimen early, thus avoiding unnecessary side effects for the patient. This research field is still relatively new and not widely known in Vietnam due to the requirement of sophisticated and expensive hardware.

With the view toward realization novel modality for breast diagnosis, we planned to apply the transillumination imaging technique noninvasively and to detect the physiological function changes in the tissue.

There are different kinds of endogenous chromophores in the biological tissue. Their absorption spectra in the optical range are sensitive to the physiological change of the animal body. In the nearinfrared region (700-1200 nm wavelength), hemoglobin has a characteristic absorption spectrum which is dependent on its redox state [8-9]. Therefore, the functional information inside the biological body can be obtained noninvasively by measuring the transmitted light [8]. By detecting the change of the absorption characteristics of the biological body, the functional change inside the body can be detected in a two-dimensional (2D) transillumination image. Using the 2D images obtained in many different orientations, 3D physiological function imaging of the biological body by transillumination imaging could be expected. This paper presents a preliminary result in the animal experiment to show the feasibility of this technique with the view toward realization of a novel imaging modality of human body parts, especially in breast tumor diagnostic imaging.

\section{Method and experimental setup}

Figure 1 shows a schematic of the experimental system to obtain transillumination images of an animal body. A male mouse (Slc:ICR, 11 week-old, 38.4g) was anesthetized by the intraperitonial injection of pentobarbital (Nembutal). The mouse was held in a cylindrical holder made of transparent acrylic resin. Laser light (Ti:Sapphire, $760 \mathrm{~nm}$ wavelength and $850 \mathrm{~nm}$ wavelength) was illuminated from one side of the holder and the image was recorded with a cooled CMOS camera (C11440-10C, 
Hamamatsu Photonics K.K.) from another side. The transillumination images were obtained while rotating the holder by rotation system. Using this technique, it would be able to reconstruct 3D image by using the filtered-back-projection algorithm if the projection images can be obtained [4].

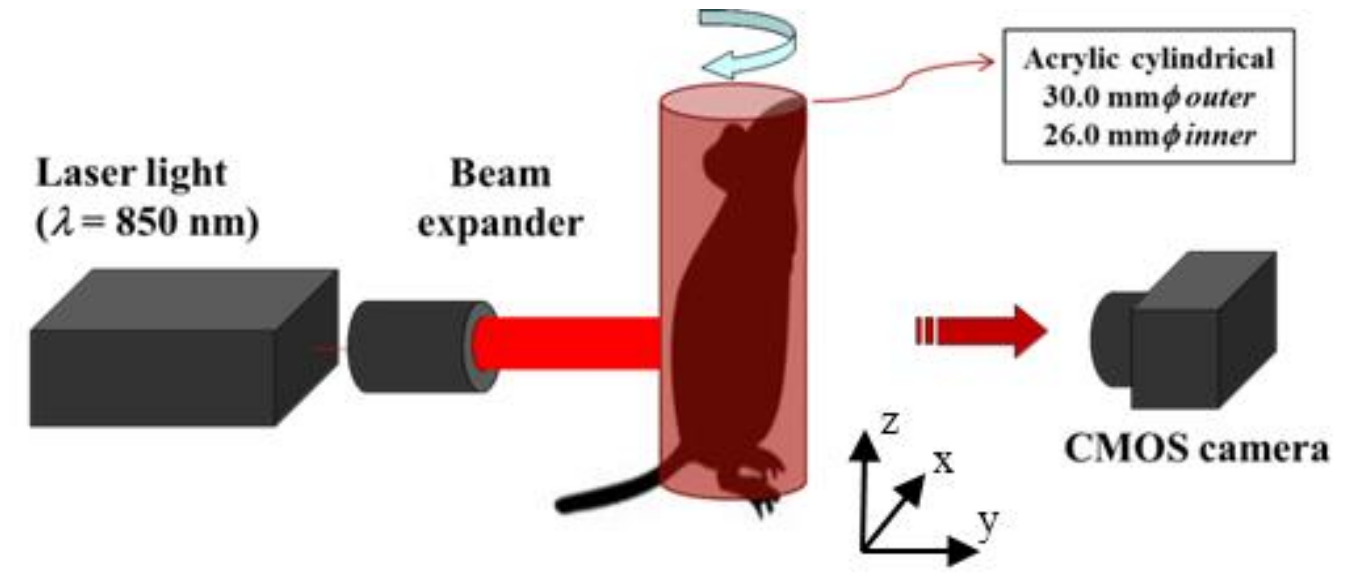

Figure 1: Experimental setup for transillumination imaging with living animal

Figure 2 show the absorption spectra of oxyhemoglobin ( $\mathrm{HbO} 2)$ and deoxyhemoglobin $(\mathrm{Hb})$ in the near infrared region. The dashed lines indicate the selected wavelengths for experiment.

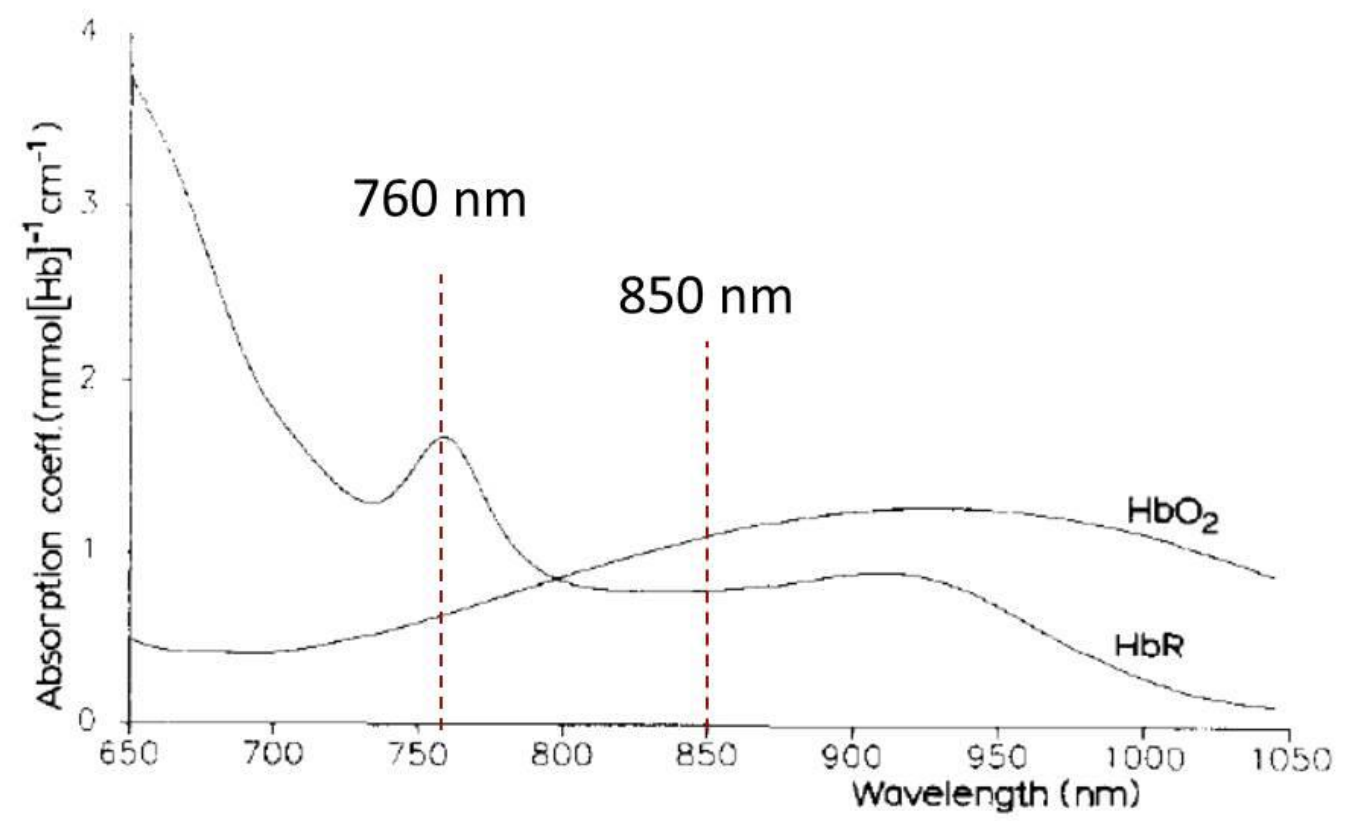

Figure 2: Haemoglobin near IR absorption spectra from lysed, normal human blood obtained from fully oxygenated and fully deoxygenated haemoglobin. (Figure adapted from [9]).

The localized change in the animal body was made by occluding the left or right blood vessel to make the blood circulation of the kidney asymmetric. Figure 3 shows an example of how to make a 
restorable occlusion of the blood vessel. A line of thread was turned around the interlobar vessel and was drawn out through a fine plastic tube. By pulling or loosening the thread, the blood vessel was occluded and opened reversibly.
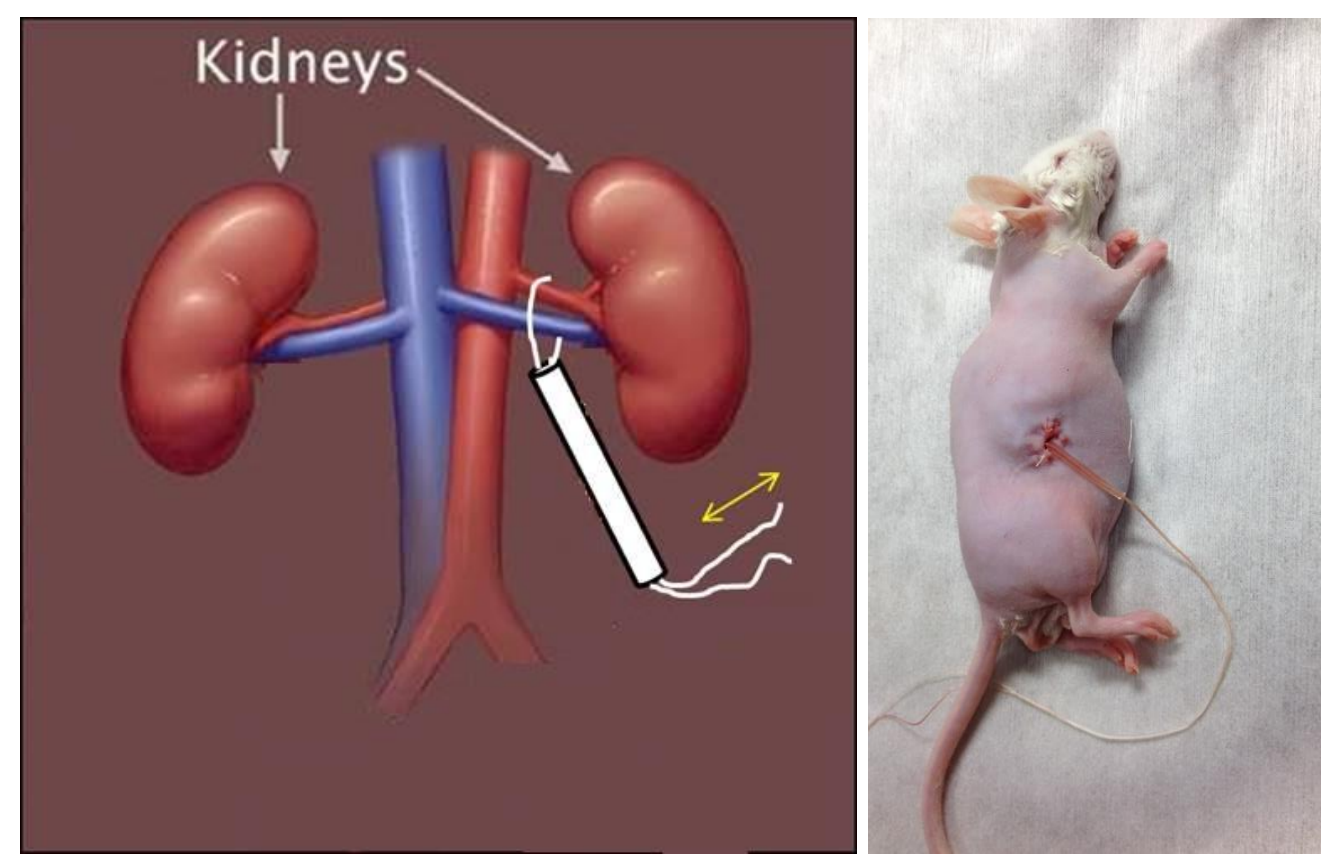

Figure 3: Control of the circulation of the kidney.

\section{Result in animal experiment}

Figures 4 and 5 show the transillumination images using a wavelength of $760 \mathrm{~nm}$. As shown in these figures, the intensity distribution is darker in Figs. 4(b) and 5(b) at the region where the thread presented, because the absorption coefficient changes by pulling the thread. While the circulation is stopped, the concentration of hemoglobin becomes higher. Therefore, the absorption coefficient is increased while using the wavelength of $760 \mathrm{~nm}$ and decreased while using the wavelength of $850 \mathrm{~nm}$. 


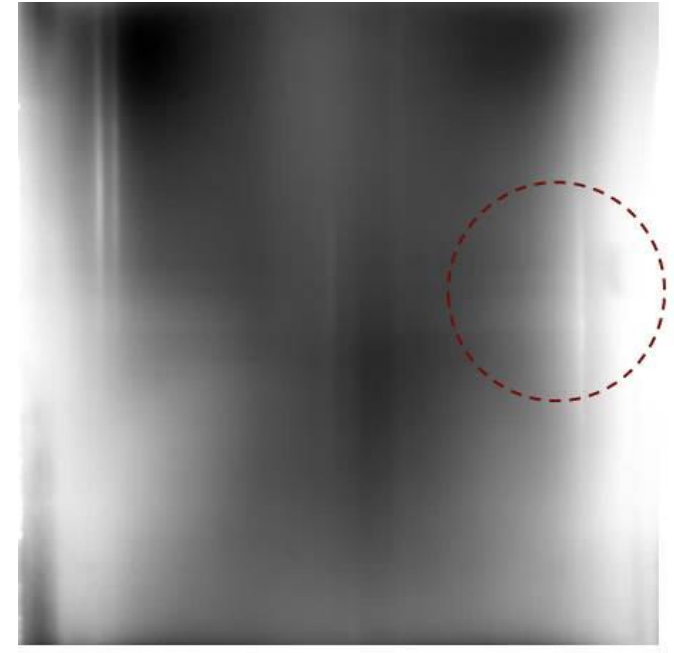

(a) Loosening the thread

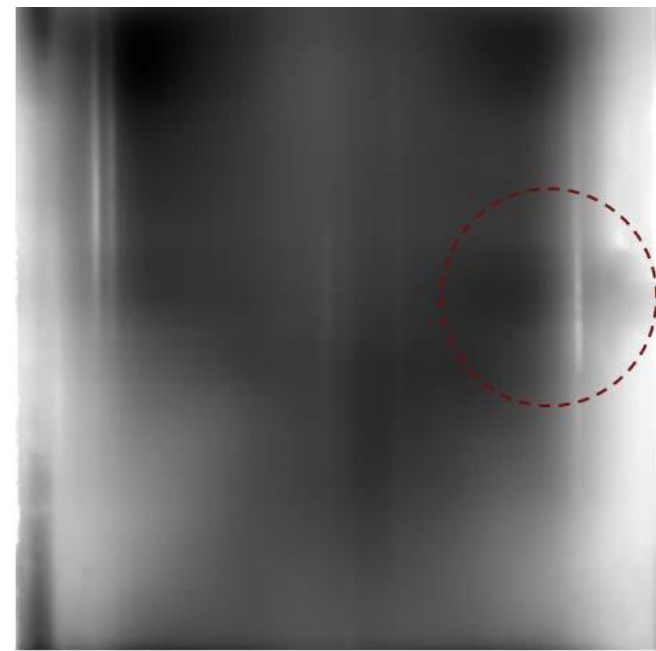

(b) Pulling the thread

Figure 4: Transillumination image of mouse's back using light source with $760 \mathrm{~nm}$ : (a). loosening the thread, (b). pulling the thread. Dashed red circle marked the region where the thread presented.

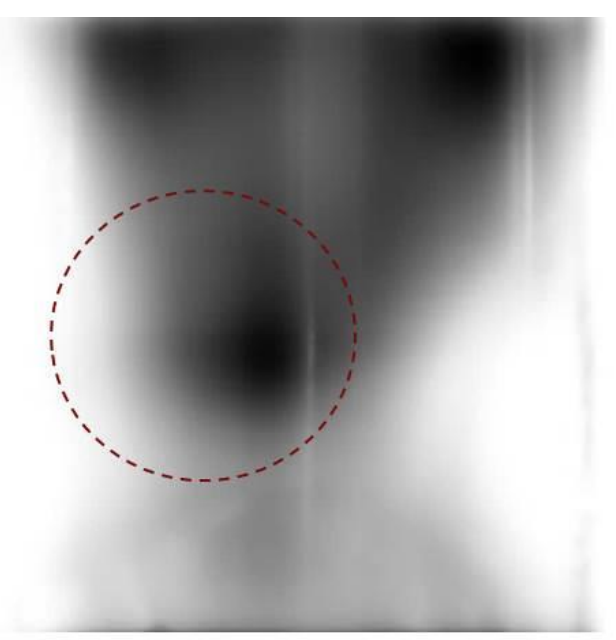

(a) Loosening the thread

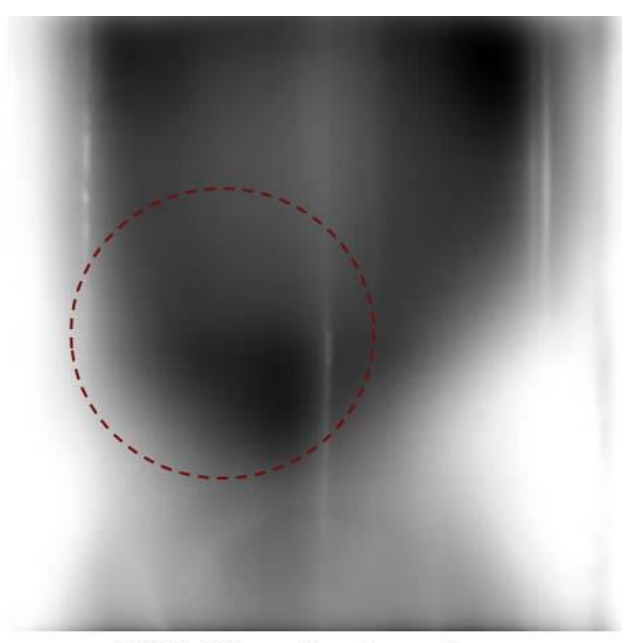

(b) Pulling the thread

Figure 5: Transillumination image of mouse's abdomen using light source with $760 \mathrm{~nm}$ : (a). loosening the thread, (b). pulling the thread. Dashed red circle marked the region where the thread presented.

By applying our 3D reconstruction technique for the unknown-structure transillumination images mentioned in the previous study [4], the cross-sectional images and 3D images were reconstructed from improved data. Figure 6 shows the cross-sectional images at the height of kidneys while loosening and pulling the thread. As shown in Fig. 6, the region where the absorption coefficient increased can be visualized. 


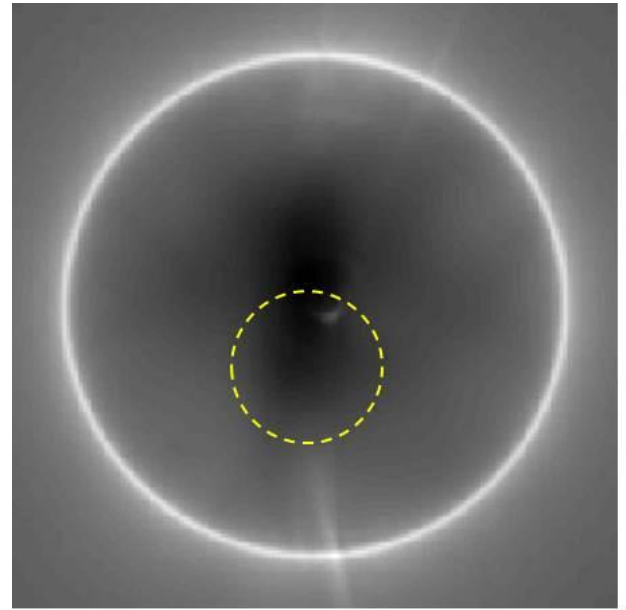

(a) Loosening the thread

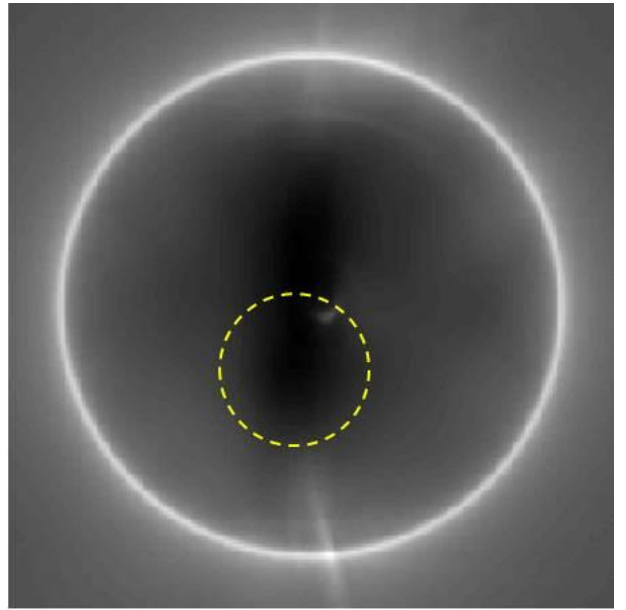

(b) Pulling the thread

Figure 6: Cross-sectional image at the height of kidneys while using the wavelength $760 \mathrm{~nm}$ : (a). loosening the thread, (b). pulling the thread. Dashed yellow circle marked the region where the thread presented.

Figure 7 shows the cross-sectional images at the height of kidneys while loosening and pulling the thread. As shown in Fig. 7, the region where the absorption coefficient decreased can be visualized.

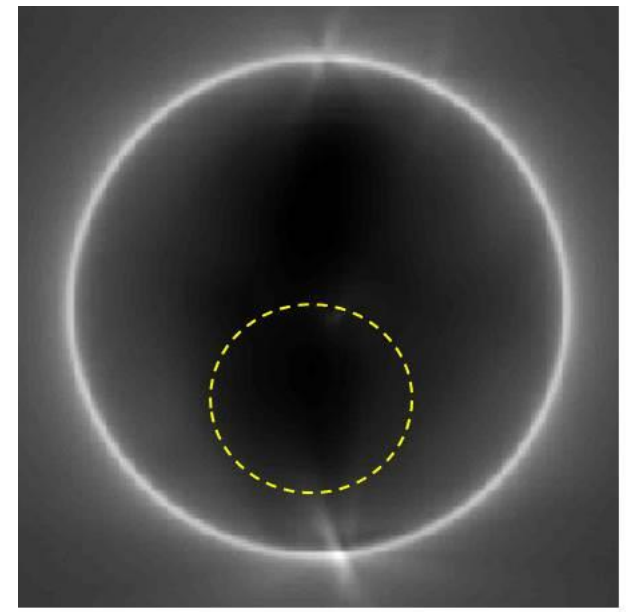

(a) Loosening the thread

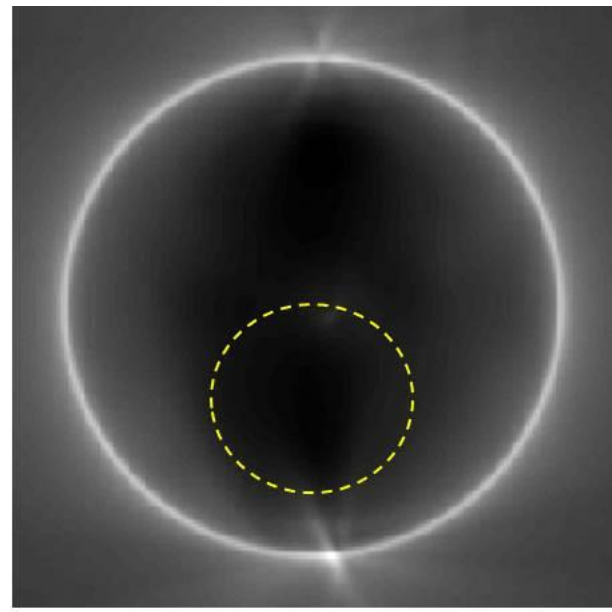

(b) Pulling the thread

Figure 7: Cross-sectional image at the height of kidneys while using the wavelength $850 \mathrm{~nm}$ : (a). loosening the thread, (b). pulling the thread. Dashed yellow circle marked the region where the thread presented.

These cross-sectional images were piled up to reconstruct the 3D image of absorbing structure in mouse body. Figures 8 and 9 respectively show the 3D image of absorbing structure in mouse body using the common threshold value with the wavelength of $760 \mathrm{~nm}$ and with the wavelength of $850 \mathrm{~nm}$. As shown in these figures, the local changes inside mouse body can be visualized. The structure was not reconstructed in successful due to the bleeding inside mouse body. However, as the local changes can be reconstructed in successful, this result suggests the possibility to realize to 3D physiological 
function imaging for small animal using transillumination image. In future work, other experiment should be done with a good skill in operation and avoided to make the bleeding as much as possible.

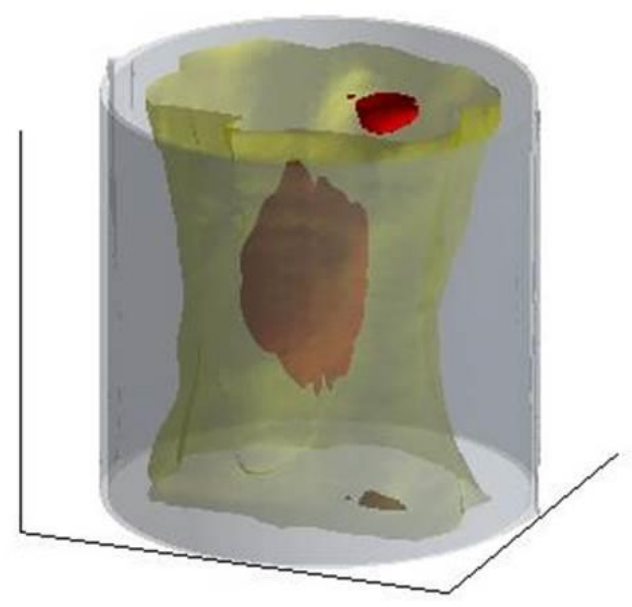

(a) Loosening the thread

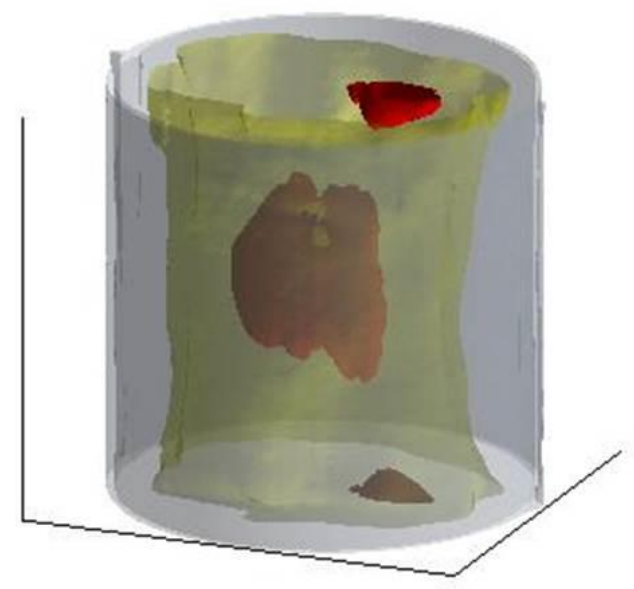

(b) Pulling the thread

Figure 8: 3D image of absorbing structure in mouse body with the wavelength $760 \mathrm{~nm}$ : (a) lossening the thread, (b) pulling the thread. The right side of each figure is the region where the thread presented.

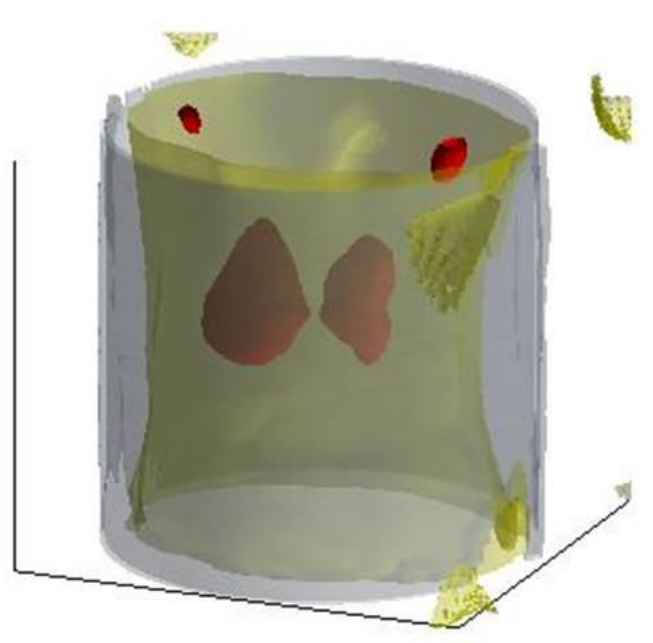

(a) Loosening the thread

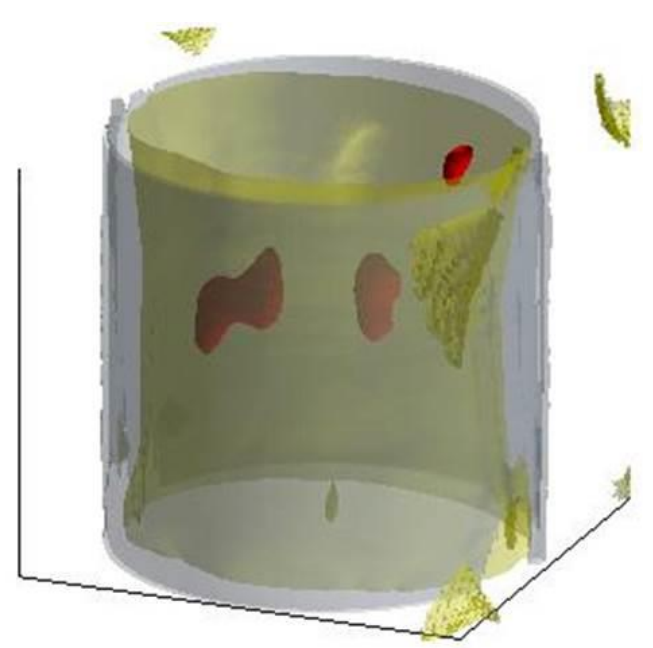

(b) Pulling the thread

Figure 9: 3D image of absorbing structure in mouse body with the wavelength $850 \mathrm{~nm}$ : (a) loosening the thread, (b) pulling the thread. The right side of each figure is the region where the thread presented.

As shown in these figures, the local changes inside mouse body can be visualized. This result suggests the possibility to realize to 3D physiological function imaging for small animal using transillumination image. In future work, other experiment should be done with a good skill in operation and avoided to make the bleeding as much as possible. 


\section{Conclusions}

With the view toward the realization of a novel imaging modality of human body parts, especially in breast tumor diagnostic imaging. We proposed the novel technique to detect physiological function changes in biological tissue using transillumination imaging. The experiment with small animal was conducted and the 3D structure of the absorbing structure was reconstructed. The possibility of the proposed technique was confirmed in the experiment. The result suggested that the information and changes of the absorbing structure in biological tissue, especially tumors in breast tissue, could be detected by changing the wavelength of the light source. These techniques are useful for developing a novel noninvasive diagnosis modality.

The animal experiment in this study was conducted in accordance with the guidance on animal research ethics is available from the World Medical Association (https://www.wma.net/policiespost/wma-statement-on-animal-use-in-biomedical-research).

\section{Acknowledgments}

This presented research was supported a part by Ho Chi Minh City University of Technology, VNUHCM under Grant T-KHUD-2018-83 to participate and present the works in the conferences.

\section{References}

[1] N. Tobisawa, T. Namita, Y. Kato and K. Shimizu, "Injection Assist System with Surface and Transillumination Images," 2011 5th International Conference on Bioinformatics and Biomedical Engineering, Wuhan, 2011, pp. 1-4.

[2] K. Shimizu, K. Tochio, and Y. Kato, "Improvement of transcutaneous fluorescent images with a depth-dependent point-spread function," Appl. Opt. 44, 2005, pp. 2154-2161.

[3] Bui P. A., Tran T. N., Tran M. T., Mô phỏng Monte Carlo sự lan truyền chùm tia laze công suất thấp trong mô ở vùng ngực và vùng chậu hông, Tạp chí Khoa học và Công nghệ, Vol. 80, 65-69, 2011.

[4] T. N. Tran, K. Yamamoto, T. Namita, Y. Kato and K. Shimizu, Biomedical Optics Express, Vol. 5, Iss. 5, 2014, pp. 1321-1335. A. Hishikawa, H. Hasegawa, and K. Yamanouchi, J. Electron Spectrosc. Relat. Phenom. Vol. 141, 2004, pp. 195-200.

[5] T. N. Tran, T. N. Phan Van, A. T. Tran, K. Yamamoto, T. Namita and K. Shimizu, Journal of Development Science and Technology, Vol. 20 - K3/2017, 2017, pp. 67-73.

[6] Zhi W, Liu G, Chang C, et al. Predicting Treatment Response of Breast Cancer to Neoadjuvant Chemotherapy Using Ultrasound-Guided Diffuse Optical Tomography. Transl Oncol. 2017; Vol. 11(1), 2017, pp 56-64.

[7] Jacqueline E. G., Emerson A. L., Hyun K. K., Molly F., Mirella A., Jessica A. C., Hanina H., Katherine D. C., Kevin K., Dawn L. H., and Andreas H. H., Dynamic Diffuse Optical Tomography for Monitoring Neoadjuvant Chemotherapy in Patients with Breast Cancer. Radiology 2018, Vol. 287:3, 2018, pp. 778-786.

[8] Y. Taka, Y. Kato, K. Shimizu, "Transillumination imaging of physiological functions by NIR light," Engineering in Medicine and Biology Society, 2000, Proceedings of the 22nd Ann. Int. Conf. of the IEEE, 2000, pp. 771-774.

[9] S. Wray, M. Cope, D.T. Delpy, J.S. Wyatt, E.O.R. Reynolds, "Characterization of the near infrared absorption spectra of cytochrome aa3 and haemoglobin for the non-invasive monitoring of cerebral oxygenation," Biochim. Biophys. Acta., Vol. 933, 1988, 184-192. 